\title{
Numerical modeling of energy-separation in cascaded Leontiev tubes with a central body
}

\author{
Maksim Makarov ${ }^{*}$ and Sofya Makarova \\ Kutateladze Institute of Thermophysics, SB RAS, 630090 Novosibirsk, 1 Lavrentiev Ave., Russia
}

\begin{abstract}
Designs of two- and three-cascaded Leontiev tubes are proposed in the paper. The results of numerical simulation of the energy separation in such tubes are presented. The efficiency parameters are determined in direct flows of helium-xenon coolant with low Prandtl number.
\end{abstract}

\section{Introduction}

The prospect of increasing the degree of gas cooling due to the cascading arrangement of energy separation tubes appeared almost simultaneously with the very idea of energy separation in a supersonic flow $[1,2]$. In one of frequent conversations about thermophysics academician of RAS A.I. Leontyev and future academician E.P. Volchkov started discussion about the effectiveness of the proposed method. It was obvious that air cooling in such devices (hereinafter referred to as Leontiev tubes) is less effective in comparison with Rank vortex tubes. However, Leontiev tubes have a significant advantage. A cooled (subsonic) part of the flow almost keeps the total pressure at the tube outlet, which allows using the cooled flow at further stages of energy separation in cascading arrangement of tubes. A significant increase in efficiency may be also achieved, using gas mixtures with low Prandtl numbers as coolants [2-4].

In this work, we propose the designs of two- and three-cascaded Leontiev tubes, present the results of numerical simulation of the effect of energy separation in such tubes, and determine the parameters of effectiveness in case of using the helium-xenon gas mixture with Prandtl number of 0.23 .

\section{Problem statement}

The main design feature of the proposed cascaded tubes of energy separation is the formation of supersonic flows in annular channels. One of the supersonic channels is formed by a profiled central body and the cylindrical inner wall of the subsonic channel. The second one is formed by a profiled outer wall of the subsonic channel and the housing. Fig. 1 presents a flow diagram in a two-cascaded tube, the main geometric and flow rate parameters, and photos of fabricated elements of a prototype sample.

For the analysis we have chosen the optimum geometry of the tube with flow characteristics that are similar to those of a single Leontiev tube, studied experimentally in

*Corresponding author: msmakarov@itp.nsc.ru 
$[5,6]$. The tube length $L$ was $450 \mathrm{~mm}$, the distance to the first critical section $\left(L_{l}\right)$ was 45 $\mathrm{mm}$, and the distance to the second critical section $\left(L_{3}\right)$ was $250 \mathrm{~mm}$. The external diameter
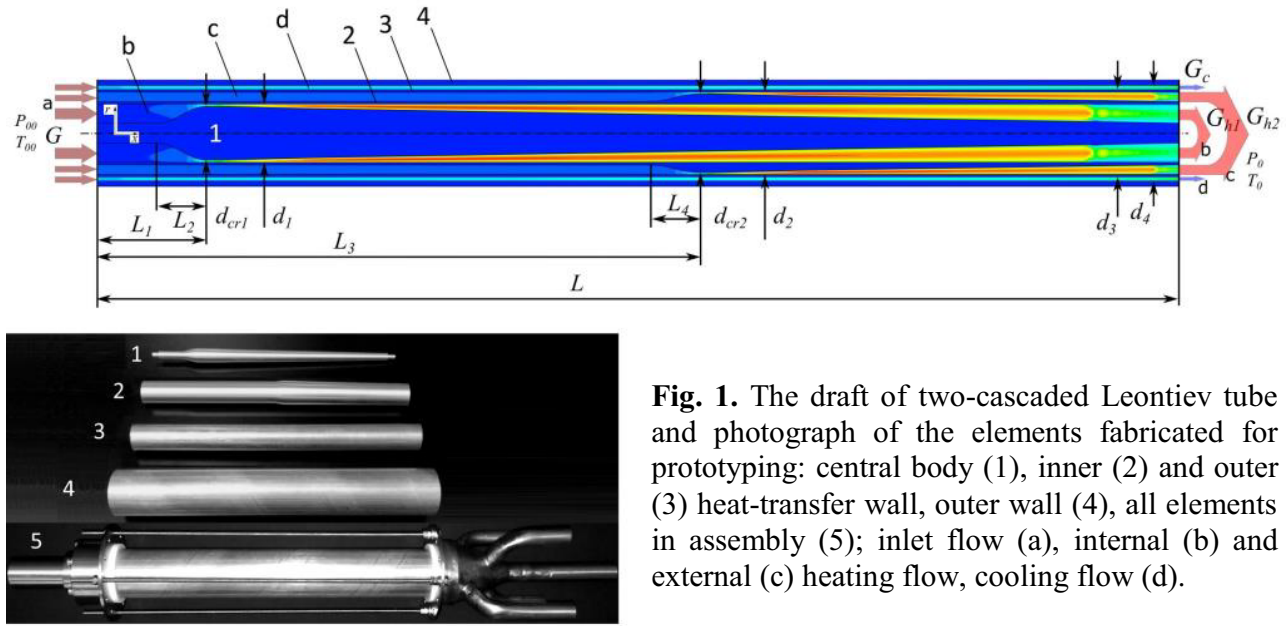

Fig. 1. The draft of two-cascaded Leontiev tube and photograph of the elements fabricated for prototyping: central body (1), inner (2) and outer (3) heat-transfer wall, outer wall (4), all elements in assembly (5); inlet flow (a), internal (b) and external (c) heating flow, cooling flow (d).

of the inner supersonic nozzle (d1) was $24 \mathrm{~mm}$, the diameter of the central body at the critical section $\left(d_{c r l}\right)$ was $22 \mathrm{~mm}$, the outer diameter of the annular nozzle with the shifted critical section $\left(d_{2}\right)$ was $34 \mathrm{~mm}$, and the outer diameter of the separation wall 4 in critical section $\left(d_{c r 2}\right)$ was $32.5 \mathrm{~mm}$. Inner and outer diameters of the subsonic channel $\left(d_{3}\right)$ and $\left(d_{4}\right)$ were $36 \mathrm{~mm} 39.5 \mathrm{~mm}$, respectively. The calculated Mach numbers at the outflow of helium-xenon mixture were 3.2 and 3.4 for central and peripheral nozzles, respectively. The results of numerical simulation taking into account the influence of viscosity have shown that the maximum Mach numbers did not exceed 2.14 and 2.33, respectively, which, as follows from previous studies, is optimal for the process of energy separation.

A three-cascaded tube has a length of $450 \mathrm{~mm}$. The critical sections are located at a distance of $45 \mathrm{~mm}, 180 \mathrm{~mm}$ and $315 \mathrm{~mm}$ from the inlet. The flow characteristics and the design modes of nozzles were selected close to the characteristics of nozzles of the twocascaded tube.

\section{Method of numerical modeling}

We solved the problem by the implicit numerical method, based on the 2D axisymmetric RANS equations for the steady compressible flow with heat transfer. ANSYS Fluent solver licensed for SSCC SB RAS and self-made code were used for mesh generation and data analysis. The S-A turbulence model and the Kays-Crawford model [7] for the turbulent Prandtl number were used for modeling. As it was obtained in [8], this numerical model allows solving the convective heat transfer problems for the turbulent low-Prandtl gas flows. The turbulent intensity was $2 \%$, and the turbulent length scale was $1 \mathrm{~m}$ at the channel inlet. The adhesion conditions for motion equations, the temperature and heat flux coupling for energy equation were set as boundary conditions on the heatconducting walls. The meshes contained from 90000 to 300000 nodes. The convergence for all variables and mass-flow rates in each channel were provided with error of $0.001 \%$.

\section{Results and discussion}

Fig. 2 shows distributions of Mach number, thermodynamic temperature, total temperature and total pressure in the longitudinal section of the two-cascaded tube for the outflow of helium-xenon mixture with the maximum obtained cooling degree of $-17{ }^{\circ} \mathrm{C}$. 
Fig. 3a presents data on the distribution of specific density of heat flux through the separating wall of the two-cascaded tube. As expected, in this configuration, the subsonic flow of a part of gas in the channel with shifted critical section (c in fig. 1) in the initial section (before critical section) is cooled by heat transfer to the central supersonic flow of another part of gas (b in fig. 1). After the critical section, the gas accelerates up to supersonic speeds and cools the third portion of gas in the outer subsonic channel ( $d$ in fig. 1). For the three-cascaded tube, the process of heat transfer from the outer subsonic channel to inner supersonic channels is the same (fig. 3b). Regardless of the number of cascades, it may be noted that in the area of interaction of subsonic flows, there is almost no heat transfer through the separation walls since the reduction temperature on the wall of the subsonic channel is little different from the initial total temperature.

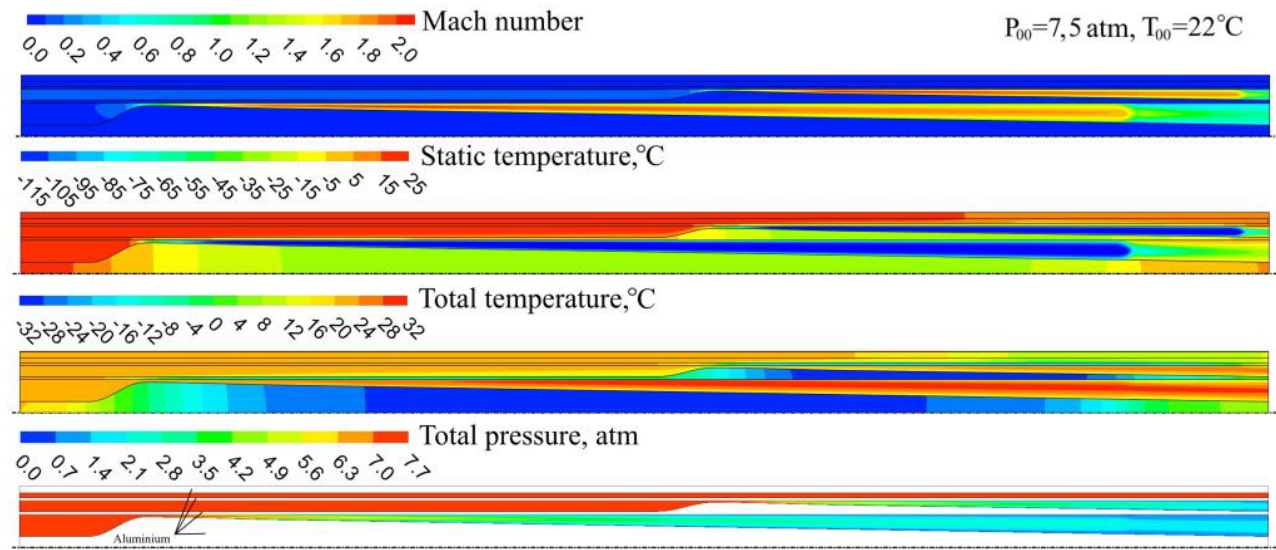

Fig. 2. Distribution of the main parameters in the longitudinal section of two-cascaded energyseparation tube with central body and separated location of critical cross-sections in annular supersonic nozzles (the data presented for the case with maximal cooling effect and $G_{C} / G^{=0.09}$ ).
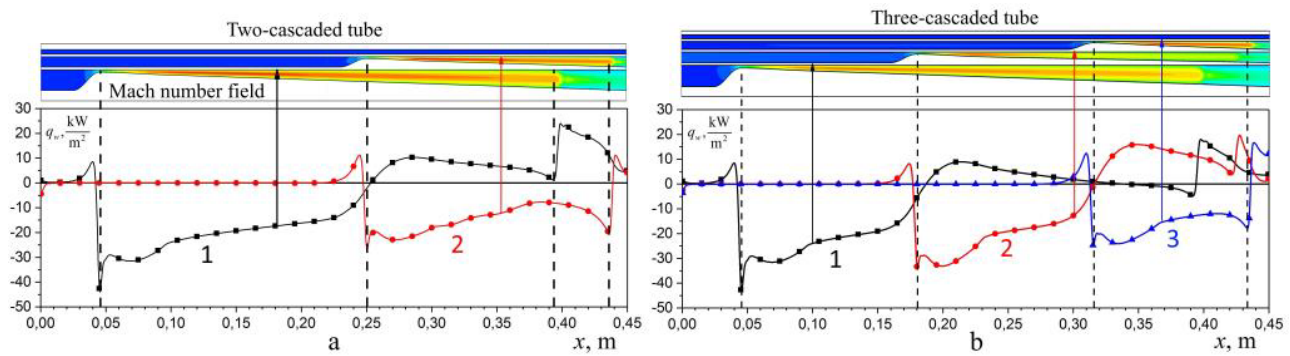

Fig. 3. Heat flux distribution at the internal heat-conducting walls for the case of two-cascaded (a) and three-cascaded (b) Leontiev tubes: data for the first cascade (1), second cascade (2), and third cascade (3) - cooling the subsonic flow; dash lines indicate critical sections of supersonic nozzles.

As can be seen in fig. 2 and fig. 3, in channels with shifted critical sections in the area of the supersonic flow, the heat flux changes its sign. Heat is transferred from the central channels to the peripheral ones, which significantly reduces the efficiency of energy separation in the cascaded tubes. This effect may be explained by the lower value of the reduction temperature in the flow, cooled in advance in the field before the critical section. The temperature of the separating wall becomes lower than the reduction temperature from the side of the central uncooled channel. Assumingly, this effect can be avoided or significantly reduced by one of the following methods. The first method is the thermal insulation of the supersonic part of the channel with shifted critical section from the side of 
the central annular nozzle. The second way is an increase in the calculated Mach numbers in central channels compared to the peripheral channels. As it turned out, without these or similar measures, the effectiveness of energy separation in the cascaded tubes of the proposed configurations does not exceed the efficiency of single tubes under the same stagnation parameters and at the same ratio of flow rates of cooled gas to the total gas flow through the tube $(\mu)$. Fig. 4 presents data on the degree of cooling, temperature efficiency factor and adiabatic efficiency [4] of energy separation in single and cascaded Leontiev tubes, depending on the ratio of cooled air or helium-xenon mixture.
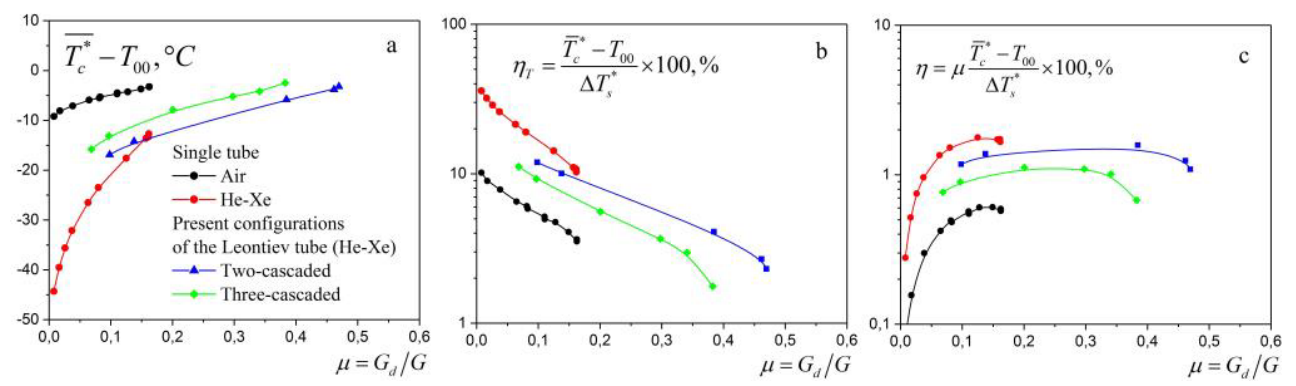

Fig. 4. Cooling effect (a), temperature efficiency factor (b) and adiabatic efficiency (c) depending on the subsonic mass flow ratio for single, two-cascaded, and three-cascaded Leontiev tubes (heliumxenon, inlet total pressure $7.5 \mathrm{~atm}$, inlet total temperature $22^{\circ} \mathrm{C}$ ).

\section{Conclusion}

Energy separation of the compressible gas flow in the two-cascaded and three-cascaded Leontiev tubes has been analyzed. The parameters of energy-separation efficiency (temperature efficiency factor and adiabatic efficiency) for the cascaded Leontiev tubes are found to be less than the same factors for the single Leontiev tube, but the studied cascaded tubes work with a higher subsonic mass flow ratio. The disadvantages of this method of energy separation in the cascaded tubes with an optimum Mach number in supersonic channels, leading to a decrease in efficiency, have been identified. Ways to address them have been proposed, in particular, by increasing the estimated Mach number from the peripheral channels to the center and by thermally insulating a part of the separating wall from the central channel.

The reported study was funded by RFBR according to the research project № 15-08-04203.

\section{References}

1. A.I. Leontiev High Temperature 351 (1997)

2. S.A. Burtsev, A.I. Leontiev Isv. AN. Energy 5 (2000)

3. M.S. Makarov, S.N. Makarova Thermophysics and Aeromechanics 206 (2013)

4. M.S. Makarov, S.N. Makarova, A.A. Shibaev JoP:CS 754 (2016)

5. Yu.A. Vinogradov, A.G. Zditovets, M.M. Strongin Fluid Dynamics 485 (2013)

6. A.G. Zditovets, Yu.A. Vinogradov, A.A. Titov IHTC15-8965 (2014)

7. B. Weigand, J.R. Ferguson, M.E. Crawford Int. J. Heat Mass Transf. 4017 (1997)

8. V.E. Nakorjakov, M.S. Makarov, Yu.I. Petukhov, O.V. Vitovsky, S.L. Elistratov, Heat transfer in a low-Prandtl gas mixtures flows (Novosibirsk, Akademizdat, 2015) [in Russian] 